\title{
Abschied von einem Freund André Weil (1906-1998)
}

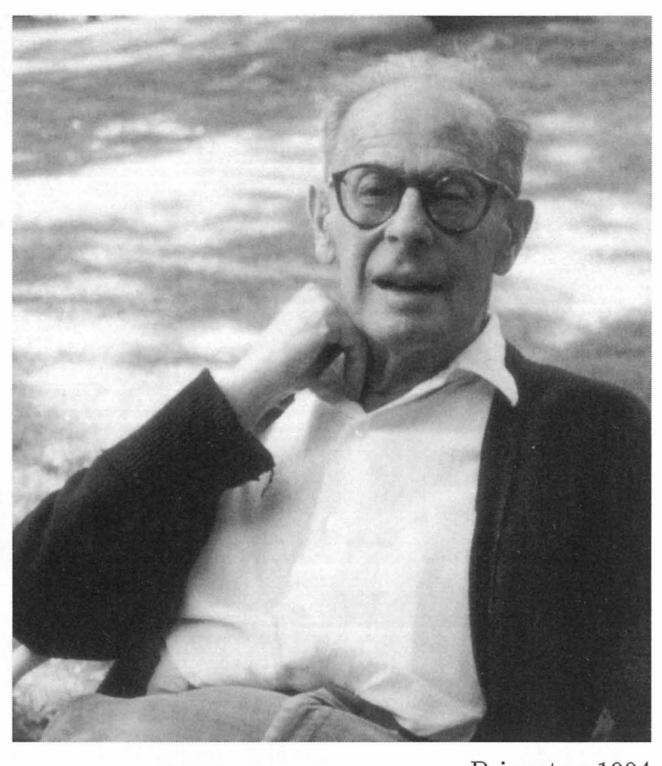

\section{von Pierre Cartier}

André Weil starb am 6. August 1998 in Princeton, im Alter von 92 Jahren. Zwei Dinge werden von ihm vor allem in Erinnerung bleiben: er war der Bruder einer berühmten Philosophin, die im Alter von 34 Jahren starb, und er war der Gründer einer berühmten Gruppe von Mathematikern mit dem seltsamen Namen Bourbaki. Ich möchte sehr persönlich über den berichten, der mein Lehrer und Freund geworden war.

André Weil hat eine Autobiographie mit dem Titel «Souvenirs d'apprentissage» veröffentlicht. Sie endet im Jahr 1947 als er zum Professor in Chicago ernannt wurde. Zu diesem Zeitpunkt hörte ich zum ersten Mal von ihm, persönlich traf ich ihn erst im Juni 1951. Danach habe ich ihn regelmäßig bis zum Ende seines Lebens wiedergesehen. Bei meinem Bericht über diese zweite Periode kann ich mich daher auf meine eigenen Erinnerungen stützen.

\section{Die Mathematik in Paris um 1950}

Die französischen Mathematiker meiner Generation sind - mit wenigen Ausnahmen - alle durch die Schmiede von Bourbaki gegangen. Henri Cartan und Laurent Schwartz waren die obligaten Meister: durch ihre Lehre an der École Normale Supérieure und am Institut Henri Poincaré, sowie durch ihre berühmten Seminare. Das Séminaire Cartan war viele Jahre lang der wöchentliche Treffpunkt der gesamten jungen Generation.

Ich hatte drei große Brüder: Jean-Pierre Serre, Armand Borel und später Alexandre Grothendieck. Samuel Eilenberg und Claude Chevalley waren ältere Freunde. Jedes Trimester versammelte sich das Séminaire Bourbaki; dort konnte man auch die Mathematiker aus der Provinz treffen, mit dem polternden Dieudonné als Anführer.

Eines der dominierenden Themen war die Gruppentheorie mit all ihren Aspekten. Die großen Namen klangen fast gleich: André Weil und Hermann Weyl. André Weil hatte 1940 sein grundlegendes Werk
"L'intégration dans les groupes topologiques et ses applications" veröffentlicht, meine Lieblingsbücher von Hermann Weyl sind „Raum, Zeit, Materie“ und "Gruppentheorie und Quantenmechanik". Weyl faszinierte durch seine Fähigkeit, sehr verschiedenartige Fäden zu verweben: Geometrie, Analysis, Algebra, Physik, Logik und Philosophie. Ein Wendehals für André Weil.

Weil und Weyl besuchten sich häufig bei zahlreichen Gelegenheiten; 1942 half der in Princeton wohletablierte Weyl vielen jüngeren Exilanten, darunter Weil, eine wenigstens vorübergehende Anlaufstelle und das Nötigste zu finden, um mehr als biologisch zu überleben. André Weil und Claude Chevalley haben in ihrem großartigen Nachruf auf Weyl die beiden oben zitierten Bücher nicht einmal erwähnt, das erfordert einige Erläuterungen.

Die Bildung von André Weil war vielseitig und nicht auf die Mathematik beschränkt. Er verehrte leidenschaftlich Literatur und Sprachen, war ein ernsthafter Liebhaber der Kunst, aber Philosophie und Physik zogen ihn gar nicht an. Seine Anregungen hat er

Wir danken Sylvie Weil für die Photographien aus ihrem Archiv. 


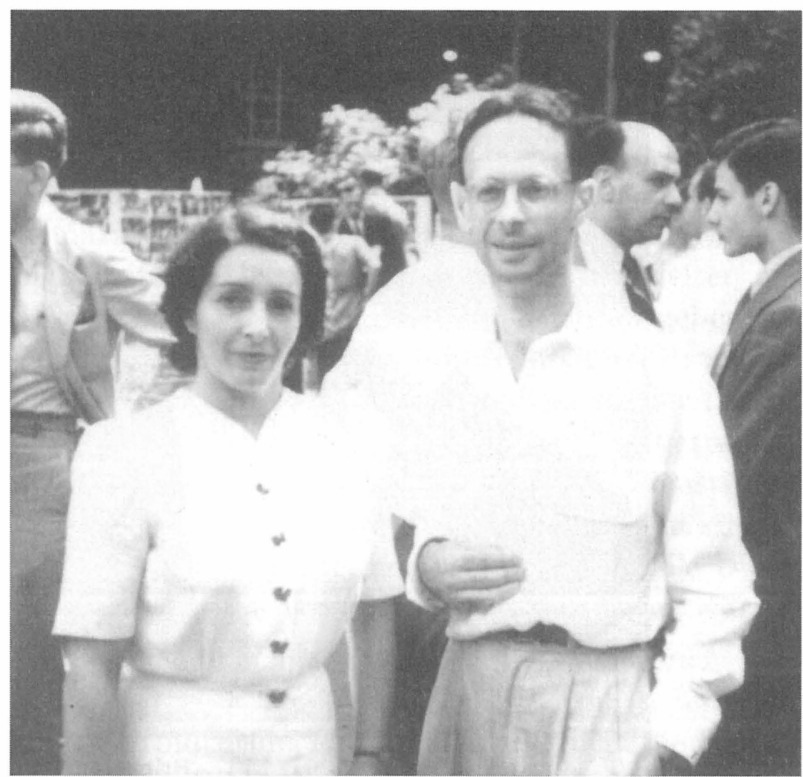

André und Eveline Weil beim ICM, Harvard 1950

niemals aus der Physik bezogen. Dieudonné und er waren sogar so verwegen, mit der Feder von Bourbaki zu behaupten, die Mathematik im 20. Jahrhundert unterläge überhaupt keinem Einfluß durch die Physik. Ich weiß, daß man solch plakative Behauptungen, die nach dem Geschmack von Weil und Dieudonné waren, etwas schattieren muß. Weil war ein Jugendfreund des Physikers Yves Rocard, der Vater des Premierministers Michel Rocard, der einer der Baumeister der nuklearen Rüstung Frankreichs war. Bourbaki hat ihn in seinen Anfängen konsultiert, um herauszufinden, welche Art von Mathematik für die Physiker nützlich sein könnte. Noch erstaunlicher war, daß Jean Coulomb, Geophysiker und späterer Direktor des C.N.R.S., bis 1939 an den Sitzungen von Bourbaki teilnahm. Seine Aufgabe sollte die Redaktion der Bände über spezielle Funktionen und Numerik sein - Projekte, die bis heute nicht realisiert sind.

\section{Die Rebellin}

Man kann nicht über André Weil schreiben, ohne seine Schwester Simone Weil (1909-1943) zu erwähnen. Es handelte sich um eine intellektuelle, geschwisterliche Verwandschaft, die so bemerkenswert war, wie die zwischen Blaise und Jacqueline Pascal. Simone war bekannter als ihr Bruder, und man konnte sicher sein, Andrés Mißmut zu erregen, wenn man ihn als Bruder von Simone vorstellte. In seinen „Souvenirs“ verweist er lediglich auf die autorisierte Biographie seiner Schwester, ohne selbst darüber etwas zu schreiben. Aber sie ist auf fast all den Fotos im Buch zu sehen, sie war bei mehreren Bourbaki-Tagungen dabei, und wer zwischen den Zeilen lesen kann, findet sie fast auf jeder Seite wieder. Als Weil 1940 im Mi- litärgefängnis sitzt, ist sie es, an die er seine Flaschenpost in Form eines Briefes schickt; dieser Brief hätte sein mathematisches Testament werden können, wie der von Galois an Chevalier, wenn alles schlecht ausgegangen wäre. Welche Zurückhaltung und Rührung in der Beschreibung von André über den Tag, an dem die Familie von Simones Tod erfährt (sie starb an Tuberkulose in einem britischen Lazarett):

[...] si par la suite son comportement m'a souvent paru, non sans raison sans doute, un défi au bon sens, néanmoins nous sommes toujours restés assez proches l'un de l'autre pour que rien de ce qui la concernait ne m'ait jamais vraiment surpris. J'en excepterais seulement sa mort, à laquelle je ne m'attendais pas, car j'avoue l'avoir crue indestructible, et je n'ai compris que fort tard que sa vie s'était déroulée suivant ses lois propres et s'était terminée de même. De sa trajectoire je n'ai guère été que lointain spectateur [...]

Im Jahr 1945 brauchten die Franzosen Helden, weil sie in Schwierigkeiten mit ihrer eigenen Identität geraten waren. Von Simone Weil, mit einem politischen Weg vom Trotzkismus zum Gaullismus, einer spirituellen Erfahrung vom Ghandismus zum Katholizismus, einer Jüdin, die sich dem Holocaust entkommen, freiwillig opferte, konnte jede Geisteshaltung etwas heroische Nahrung beziehen.

\section{Der Verbannte}

Die Lektüre der „Souvenirs" offenbart zwei wesentliche Erfahrungen, die Weils erste Lebenshälfte entscheidend beeinflußten, nämlich seine Reise nach Indien und die Drangsale während des Krieges von 1939-1945. André und Simone schöpften aus den selben Quellen: der Lektüre der Bhagavādgit $\bar{a}$, dem Dichter Rabidranāt Tāgore und vor allem aus den Kampagnen zivilen Ungehorsams von Ghandi. Auf diese philosophische Basis gründet er seine Einstellung zum drohenden Krieg: er reist in ein neutrales Land und wandert dann in die USA aus. Als neutrales Land wählte er Finnland; aber er mußte zugeben, daß die Wahl dieses zwischen Hitler und Stalin eingekeilten Landes nicht die klügste war. Die mathematischen Fähigkeiten von Weil mußten bewahrt werden, "pour l'honneur de l'esprit humain" in den Worten von Jacobi. Einige haben dies in boshafter Weise interpretiert als „mein Gehirn ist zu wertvoll, um den Kugeln ausgesetzt zu werden“ oder „am Krieg teilzunehmen würde mich unwiederbringliche Zeit kosten, da ich schwanger bin von großartigen Entdeckungen". Sie wollten dabei nur den sehen, der in Krisenzeiten mit der "Moral eines reinen Mathematikers" seinen Egoismus offenbart hat. In unseren Kreisen ist das recht verbreitet und dient oft als Alibi, der Verantwortung aus dem Weg zu gehen. 


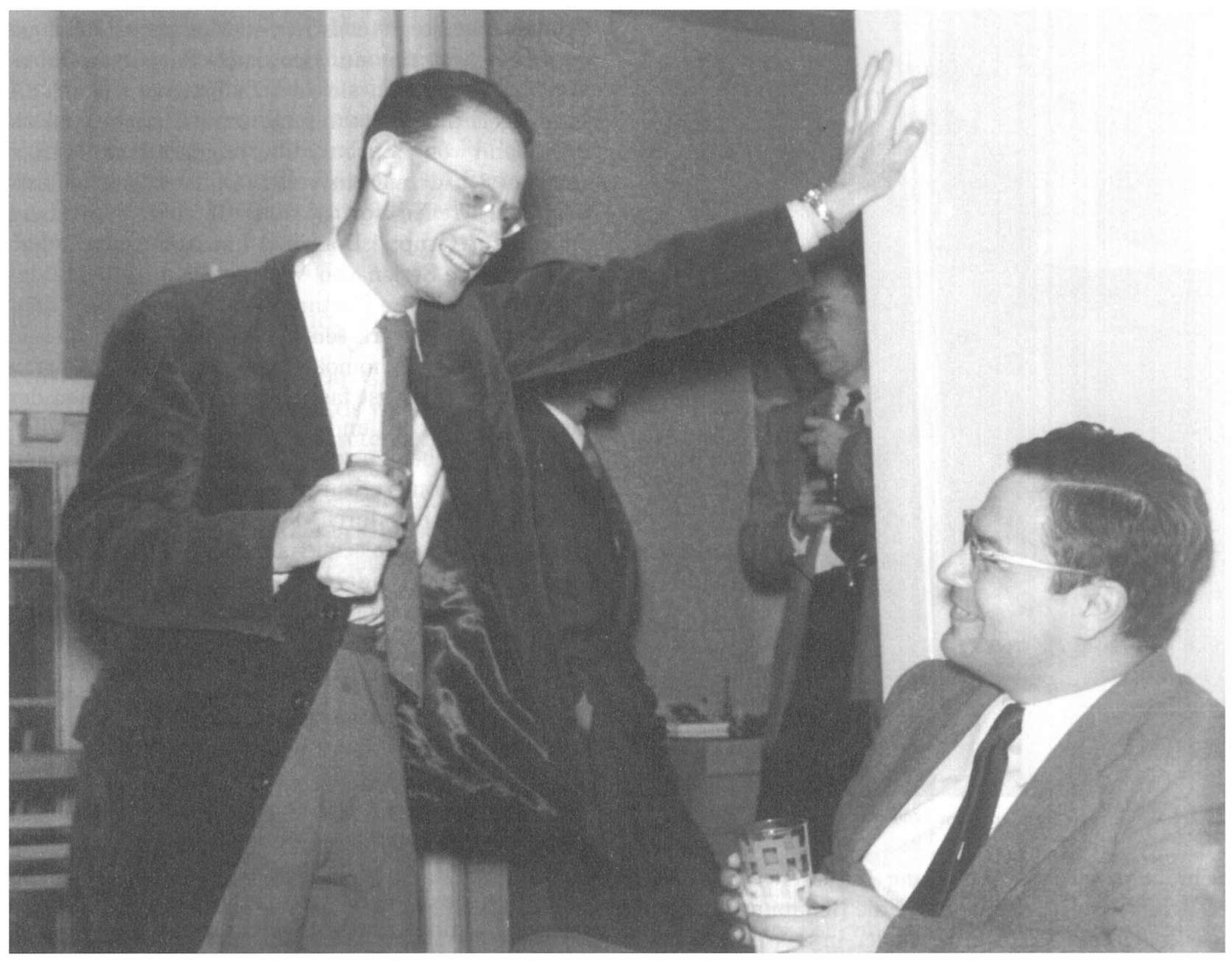

André Weil und Armand Borel, Chicago um 1955

Die wichtigste Ursache für Andrés unpolitische Haltung ist Simones Engagement, das ihm maßlos erschien. Aber man muß auch Weils familiäres Milieu in Betracht ziehen: Seine Mutter ist eine österreichische Jüdin, geboren in Rußland, dann nach Belgien und Frankreich emigriert, um den zaristischen Pogromen zu entkommen. Sein Vater ist ein elsässischer Jude, ausgebildet im Gymnasium und an der Universität des zu der Zeit deutschen Straßburg. Er leistet seinen Militärdienst in Deutschland, bevor er sich für Frankreich entscheidet und dann von 1914-18 auf der französischen Seite als Arzt am Krieg teilnimmt. Seine Tante mütterlicherseits lebt bis zur Nazizeit in Frankfurt und er besucht sie häufig. In seiner Familie wurde vermutlich fast genausoviel deutsch wie französich gesprochen. Mein Familienkreis war etwas ähnlich, und man muß verstehen, daß wir Franzosen, aufgewachsen mit deutscher Kultur, und Juden mit kosmopolitischer Neigung, aufgerufen waren, das Deutschland Hitlers im Namen des Deutschlands von Goethe zu bekämpfen.
Weil hat sich über die Art, in der man ihn während der vier Kriegsjahre (1941-45) in den USA aufgenommen hat, oft beklagt. Es ist richtig, daß er nur bescheidene Mittel zum Überleben hatte, und daß er in einem ziemlich miserablen College unterrichten mußte, während es für Princeton oder Harvard eine Ehre gewesen wäre, ihn anzuwerben. Nach den Erzählungen von Exilanten wie dem Mathematiker Hochschild oder deutschen Freunden meiner Mutter mußten die Flüchtlinge aus Europa oft einen Kreuzweg gehen, und die Zeugnisse von Antisemitismus an den amerikanischen Universitäten um 1940 könnten Bände füllen. Aber schließlich muß ich doch zu bedenken geben, daß Weil nach seiner Ankunft in New York im März 1941 von direktem Kontakt zum Krieg verschont war, ruhig, wenn auch bescheiden leben, und eines seiner wichtigen Bücher schreiben konnte: „Foundations of Algebraic Geometry“. Pardon André, aber Deine Schwester sah das klarer: der Krieg gegen Hitler war sehr wohl unser Krieg. 


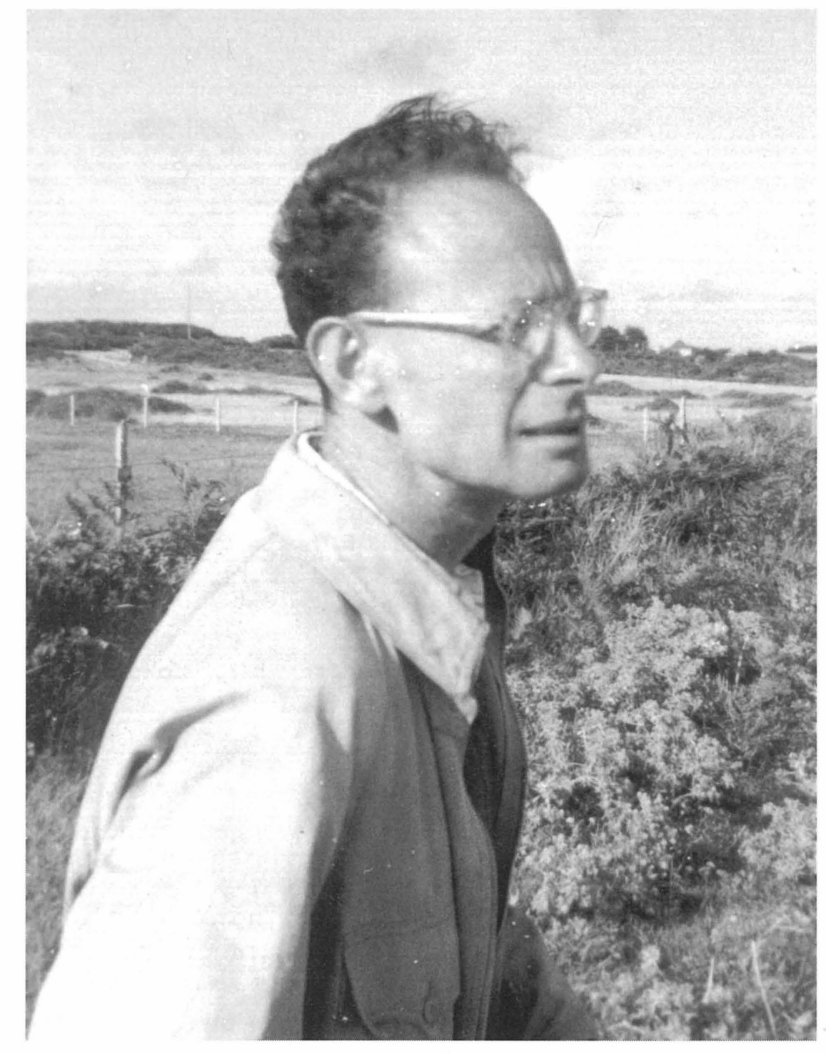

André Weil in der Bretagne, um 1960

Sehr bald nach der Befreiung Frankreichs im Jahr 1944 hat sich Henri Cartan für die Rehabilitation von Weil eingesetzt. Er wurde wieder in das französiche System integriert, und hatte eine fiktive Stelle als „détaché à l'étranger". Von Januar 1945 bis September 1947 war er Professor in São Paulo (Brasilien), zusammen mit Zariski und Dieudonné. Als ich ihn 1951 kennenlernte, hatte er schon eine Position, die auf ihn zugeschnitten war: er verbrachte neun Monate im Jahr an der Universität Chicago und war frei, jedes Frühjahr nach Paris zurückzukommen. Das blieb so bis 1958, als er zum Professor in Princeton ernannt wurde; dort blieb er bis zum Ende seiner Laufbahn.

\section{Bourbaki und die Zauberflöte}

Ich sehe die Bücher von Bourbaki gerne als eine Art von Zauberflöten, die den jungen Taminos ausgehändigt werden, damit sie den Zugang zur wahren Mathematik finden. Weil würde ich die Rolle des Sarastro übertragen, des unendlich klugen und wissenden Magiers, zugleich angsteinflößend und wohlwollend. Er war der Herr des Prüfungstempels. Aber genug davon!

Im Herbst 1950 wurde ich Schüler der École Normale. Für die Mathematik war dies ein gesegnetes Jahr:
Samuel Eilenberg - er ist einige Monate vor Weil gestorben - war für ein Jahr nach Paris eingeladen. Weil und er hatten sich als Exilanten in den USA kennengelernt; Weil wünschte seine Mitarbeit an einem Band von Bourbaki über algebraische Topologie, die sich damals in vollem Aufschwung befand. Seit dem Treffen von Oktober 1950 war Eilenberg offiziell zur Gruppe Bourbaki hinzugewählt. Er gab dort mehrere Serien von Vorträgen und drückte damit dem Séminaire Cartan dieses Jahres - dem dritten der Serie - stark seinen Stempel auf. In diesem Jahr entstand die homologische Algebra von Cartan und Eilenberg. Es ist faszinierend aus den Noten der Séminaires Cartan und Bourbaki den funktoriellen Zugang entstehen zu sehen, der später von Grothendieck perfektioniert wurde. Leray hatte meisterhaft den Weg geebnet durch die Erfindung der Begriffe Garbe und Spektralsequenz - er erhält dafür nicht immer die gebührende Anerkennung. Das eigentliche Genie von Bourbaki - hier verkörpert durch Cartan, Eilenberg und Serre - bestand darin, die grundlegenden Begriffe herauszuarbeiten, und damit alles zu vereinfachen.

Ende der fünfziger Jahre begann der Siegeszug von Bourbaki. Er steckte sie nacheinander alle in seinen Sack: das Collège de France, die Sorbonne, natürlich die Écoles Normales, danach die École Polytechnique und den C.N.R.S., und schließlich - oh Schreck - die Académie des Sciences. Der Jungtürke hat sich das wissenschaftliche Establishment einverleibt.

Bourbaki war ohne Zweifel die Schöpfung von Weil, wenngleich es nicht an anderen starken Persönlichkeiten mangelte. Bei Bourbaki herrschte Begeisterung und ein offener Ton. Die Art von Weil, gerne zu streiten, verbunden mit der „Moskitotaktik" der kleinen Stiche von Cartan und der überschäumenden Energie von Dieudonné, führten oft zu ritualisierten Psychodramen.

Am 4. Juli 1956 traf sich Bourbaki zu seinem Sommerkongreß von zwei Wochen in Die (Drôme), man feierte an diesem Tag bei guter Laune den 50. Geburtstag von Dieudonné. Während des Nachtischs und bei den Trinksprüchen verlas Cartan einen Brief des nicht anwesenden André Weil. Er erinnerte daran, daß sich die Gründer damals verpflichtet hatten, mit 50 Jahren aufzuhören. Er selbst hatte dieses Alter nun erreicht und er erklärte seinen Rücktritt. Als Gründe nannte er zum einen die Schwierigkeit, eine Gruppe von 17 Mitgliedern nach den vereinbarten Regeln arbeitsfähig zu erhalten, und zum anderen die Notwendigkeit, der jüngeren Generation die Übernahme ihrer Verantwortung zu ermöglichen. Dieser Brief rief Bestürzung und Betroffenheit hervor, trotzdem nahm man Weils Rücktritt an. Die anderen Gründer zogen jedoch nicht mit und wollten 
noch fünf oder sechs Jahre bis zu ihrem Ausscheiden abwarten. Weil selbst nahm noch gelegentlich an Bourbaki-Kongressen teil; ich erinnere mich an eine denkwürdige Auseinandersetzung zwischen Weil und Grothendieck im Jahr 1960 in Pelvoux-le-Poët und an die Lektüre einer ersten Version der „Basic Number Theory" von Weil im Jahre 1964 oder 65 in der Nähe von Grasse.

\section{Das Orakel von Princeton}

1957 gewährte die Universität Chicago André Weil ein „Sabbatical“, das er in Paris verbrachte, als gerade die Kolonisierung des Institute for Advanced Study (I.A.S.) durch Bourbaki begann. Armand Borel war dort 1957 zum Professor ernannt worden, und Serre verbrachte jeden zweiten Herbst am I.A.S. Im Herbst 1958 erhielt auch Weil eine permanente Stelle. Er blieb dort bis zu seiner Emeritierung im Jahr 1976, und verließ Princeton danach nicht mehr.

Von dem sarkastischen Freeman Dyson, auch einem permanenten Professor der Physik am I.A.S., habe ich einmal folgende Beschreibung von Princeton gehört: „A nice place to dry up." Es ist nur allzu richtig, daß das I.A.S. lange Zeit ein Nebenolymp war, bevölkert von Halbgöttern, die ihre kreative Zeit hinter sich hatten, mit einem Hühnerhof von PostDocs, die gekommen waren, um sich eine Atempause von ein oder zwei Jahren zu gönnen, bevor sie sich in den Universitätsalltag stürzen mußten. Als ich 1957, einige Jahre nach dem Tod von Einstein, für zwei Jahre dort ankam, gab es da eine schöne Sammlung alter Elefanten. Die Mathematiker Whitney und Selberg kamen wie zu einem Ritual am späten Vormittag, um Zeitung zu lesen, und es war sehr schwierig, mit ihnen ernsthaft zu diskutieren. Gödel war ein flüchtiger Schatten, schon ganz Beute seiner Paranoia. Glücklicherweise gab es auch Borel, Weil und bald Harish-Chandra; jeder war auf seine Weise anregend und die jungen Gäste wie ich konnten von ihnen profitieren.

André Weil verwandelte sofort das Séminaire Hadamard seiner Jugend in ein „Joint Institute-University Seminar on current literature“, es überlebte bis 1962. Fast jedes Jahr hielt er eine Vorlesung am I.A.S. Seine Interessen kreisten um Zahlentheorie, es waren nacheinander

a. Adele und algebraische Gruppen, Anwendungen auf diskrete Untergruppen von Liegruppen

b. Automorphe Funktionen und Dirichletreihen

c. Geschichte der Zahlentheorie.

Weil war nicht das, was man einen ,großen Prediger" nennt, ich spreche aus Erfahrung als einer der

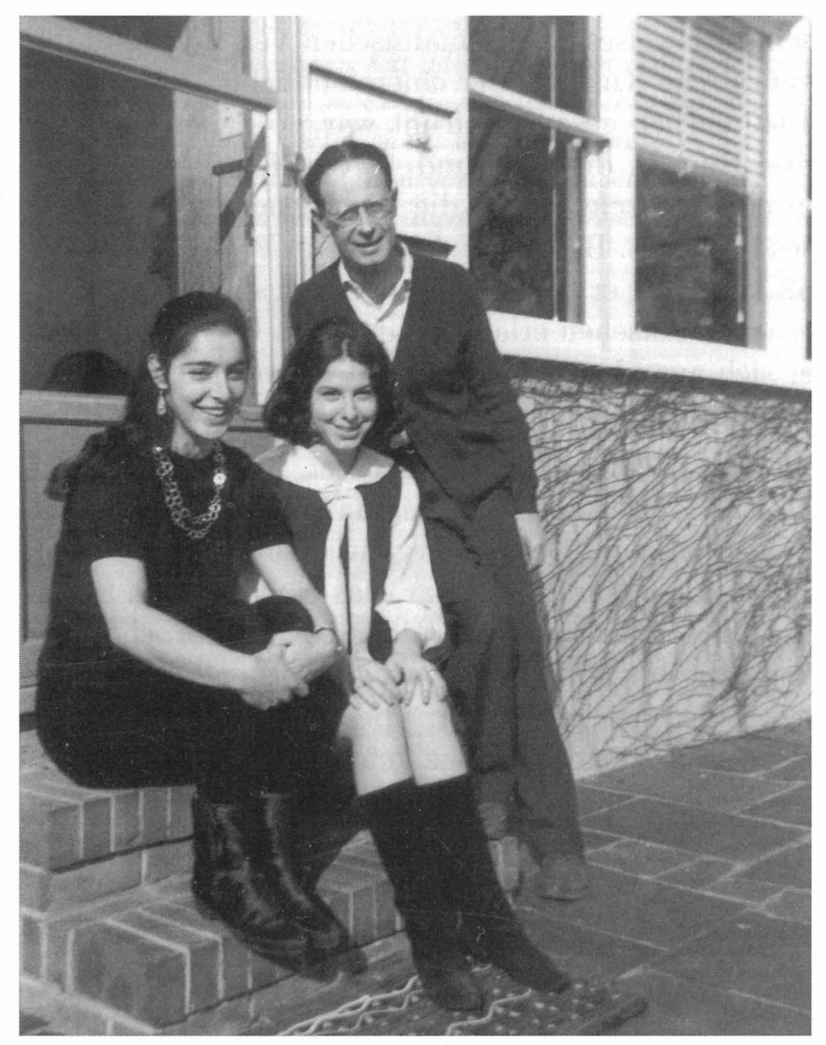

André Weil mit seinen Töchtern Sylvie (links) und Nicolette, Princeton 1966

nicht zahlreichen unerschrockenen Hörer seiner Vorlesungen. Der Stil, in dem er Mathematik aufschrieb, war oft schwerfällig, und erreichte nicht die sarkastische Eleganz seiner „Souvenirs“. Er konnte sich leicht in endlosen Rechnungen ergehen und machte sich einen Höllenspaß daraus, das wesentliche erst ganz am Schluß zu verraten.

In gewisser Weise verlief der Beginn seiner mathematischen Karriere ziemlich langsam - wenn man von all dem absieht, was er fast anonym in die Unternehmung Bourbaki eingebracht hat. Bei seiner Rückkehr aus Indien war seine Bilanz - gemessen mit heutigen Maßstäben - recht bescheiden. Mit 26 Jahren hatte er eine Thèse von 30 Seiten und einigen Kleinkram veröffentlicht, der nichts mit seinen wahren Interessen zu tun hatte, die sich nur um die Zahlentheorie drehten. Er hat versichert, daß seine Thèse damals nur von ein oder zwei Leuten gelesen worden war, und daß sie Lücken enthielt, die ein hochgradig inkompetenter Referent nicht entdeckt hatte. 70 Jahre später muß man sagen, daß die fragliche Thèse wegen ihrer Neuartigkeit und Tiefe genügt hätte, hohes Ansehen zu erwerben und eine mehr als ehrenwerte wissenschaftliche Laufbahn zu begründen. Sein Buch über die Integration in Gruppen wurde 1935 geschrieben und 1940 veröffentlicht, seine Wirkung entfaltete es erst nach 1945. Wenn auch die Ankündigung 
seines Beweises der Riemannschen Vermutung für algebraische Kurven über einem endlichen Körper von 1940 ehrlich gesagt verfrüht war, so haben doch die drei Bücher, die 1946 und 48 veröffentlicht wurden, in einwandfreier Weise die Richtigkeit seines Beweises bestätigt. Bei seiner Ankunft in Princeton war er 52 Jahre alt, er hatte inzwischen ein solides und dauerhaftes Ansehen erlangt; auf diesen Lorbeeren hätte er sich ausruhen können, er tat es aber nicht.

André Weil war gewiß kein einfacher Mensch, sein Spott war beißend und gefürchtet. Ich erinnere mich an einige Episoden zu Beginn seiner Tätigkeit in Princeton: zum Beispiel organisierte Borel in seinem Büro ein geheimes Seminar, das einen ganzen Samstag lang von 9 bis 18 Uhr dauerte, unterbrochen nur von einer kurzen Mittagspause. Grothendieck behandelte das gleiche Thema, über das Leray früher in Princeton einige Vorträge gehalten hatte, die Theorie der Residuen, aber im Gegensatz zum Analytiker Leray behandelte er es vom rein algebraischen Standpunkt aus. Weil gab mit sehr lauter Stimme den folgenden Kommentar: „Wir müßten unsere Kollegen von der Physik bitten, ein Prinzip der Antiinterferenz zu erfinden, die zwei Dunkelheiten (Grothendieck und Leray) zu Licht erstrahlen läßt." Der Bruch zwischen Weil und Grothendieck fand zwei Jahre später bei dem schon erwähnten Bourbaki-Kongreß statt.

Das ist nicht die einzige Gelegenheit, bei der Weil sich von der Polemik hinreißen ließ. Was war eigentlich der Auslöser des Bruchs zwischen Weil und seinem ehemaligen Schüler Serge Lang? Die beiden waren eben Meister in gegenseitigen Beschuldigungen. Anscheinend war Weil ein verzogenes Kind, und vielleicht hatte er eine Fortsetzung der endlosen Streitereien mit Simone im Sinn, wo Bruder und Schwester sich wie wild bissen, ohne sich wirklich zu verletzen.

\section{Die letzten Jahre}

Weil ging 1976 in Ruhestand. Er blieb in Princeton, da sich seine Familie inzwischen an den Nordosten der USA gewöhnt hatte. Bis kurz vor seinem Tod hielt er an der alten Gewohnheit fest, das Frühjahr in Paris und den Sommer im Departement Mayenne $\mathrm{zu}$ verbringen. Er veröffentlichte noch einige Arbeiten, vor allem über Geschichte der Mathematik, aber sein Meisterstück besteht aus zwei Büchern: das erste „Elliptic Functions according to Eisenstein and Kronecker" ist eine Neufassung von Eisenstein, durch die er zeigte, daß er der beste der modernen Zahlentheoretiker war. Man kann dieses Buch schon als Fragment der Zahlentheorie des XXI. Jahrhunderts ansehen. Das zweite - "Number Theory" - ist aus seinen letzten Vorlesungen entstanden, wo er sich vorgenommen hatte, von Fermat bis zur gegenwärtigen Forschung fortzuschreiten. Er erklärt dort, was ihn bei der Lektüre von Fermat und Euler inspiriert hat. Für ihn bedeutete Geschichte der Mathematik in erster Linie, die Klassiker zu lesen und sich dadurch inspirieren zu lassen. Außerdem gab er seine „CEuvres Scientifiques" in drei Bänden heraus, und verfaßte zu jedem Band einen außerordentlich reichhaltigen Kommentar, der eine intellektuelle Autobiographie ist.

Ich war selbst Zeuge sehr lebhafter Szenen mit André Weil gewesen, bei Bourbaki und im täglichen Leben. Ich habe gesehen, wie Kleinigkeiten - eine leichte Verrenkung oder ein Essen, das nicht nach seinem Geschmack zubereitet war - für ihn epische Ausmaße annahmen. Dagegen beschreiben die „Souvenirs" ein recht ausgeglichenes Wesen, jedenfalls versöhnt. War er in den letzten Jahren anders geworden, wenn man von seinem Auftreten in der Öffentlichkeit etwas absieht?

In den zwei letzten Jahren ließ er sehr nach und fühlte sich einsam. Die Prüfungen des Endes blieben ihm weitgehend erspart, er starb plötzlich. Er glaubte nicht mehr als ich an ein Überleben nach dem Tod, aber sein Werk wird ihn wenigstens für einige Zeit überdauern. Bei seiner Gründung von Bourbaki zur Förderung der Einheit der Mathematik glaubte er an einen Euklid des 20. Jahrhunderts.

\section{Biographische Literatur}

[1] S. Pétrement: La vie de Simone Weil. Fayard 1978

[2] A. Weil: Souvenirs d'apprentissage, Birkhäuser, Boston 1991 (Deutsche Übersetzung: Lehr- und Wanderjahre eines Mathematikers. Birkhäuser, Basel 1993)

Aus dem Französischen von Gerd Fischer

\author{
Adresse des Autors \\ Prof. Pierre Cartier \\ École Normale Supérieure \\ 45, rue d'Ulm \\ F 75230 Paris Cedex 05 \\ cartier@ihes.fr
}

